\title{
Urgences
}

\section{Posthumus : la succession " desarroyée " de Ferron}

\section{Ginette Michaud}

Numéro 23, avril 1989

Lisière du livre

URI : https://id.erudit.org/iderudit/025517ar

DOI : https://doi.org/10.7202/025517ar

Aller au sommaire du numéro

Éditeur(s)

Urgences

ISSN

0226-9554 (imprimé)

1927-3924 (numérique)

Découvrir la revue

Citer cet article

Michaud, G. (1989). Posthumus : la succession " desarroyée " de Ferron.

Urgences, (23), 58-67. https://doi.org/10.7202/025517ar d'utilisation que vous pouvez consulter en ligne.

https://apropos.erudit.org/fr/usagers/politique-dutilisation/ 


\section{GINETTE MICHAUD}

\section{Posthumus: la succession «desarroyée» de Ferron}

Mon titre indique déjà ce qui retiendra ici mon attention: profitant de l'oscillation de sens que m'offre le mot «posthume" à propos duquel Gérard Genette fait remarquer qu'il ne signifie pas, comme le laisserait croire une "très ancienne et (superbe) fausse étymologie», «postérieur à la mise en terre», mais bien, tout simplement, puisqu'il est le superlatif de "posterus'" , ce qui voit le jour ou vient après la mort de quelqu'un -, je voudrais interroger, à partir de deux livres de Jacques Ferron récemment publiés par VLB éditeur, La conférence inachevée ${ }^{2}$ et Le désarroi ${ }^{3}$, certains effets relevant d'une stratégie éditoriale qui n'est pas sans influencer, sinon contraindre, notre façon de lire ces textes. Car il faut se demander ce qui est engagé par une telle stratégie ${ }^{4}$ qui, opérant nécessairement dans l'après-coup, exerce sur les textes posthumes un pouvoir peut-être plus manifeste que sur les oeuvres publiées du vivant de leur auteur. Cette ingérence, cette gérance aussi, se signalent, entre autres traits, par une présence indue qui tente de réapproprier ces suppléments (il s'agit de fait souvent de brouillons, mais ce n'est pas le cas ici), de concerter - c'est-à-dire arranger, combiner, organiser: tout le contraire du désarroi, mot qui vient du verbe "desarroyer, desareer", mettre en désordre - ces restes à l'intérieur du projet prémédité de l'oeuvre. La tâche de la succession étant d'assurer l'enchaînement, l'ordre, la suite, elle cherchera donc à orienter ces textes derniers vers les commencements de l'oeuvre et, les retournant de la sorte vers leurs origines, à assurer sa circularité et sa clôture, bref, à boucler la boucle. On retrouve alors le déplacement, le glissement de sens, précisément, que j'évoquais à l'instant et qui fait passer de la vraie à la fausse étymologie du "posthumus", c'est-à-dire à la manipulation, même faite discrètement, en sous-main ${ }^{5}$ comme ici, qui consiste à faire de tels textes le lieu d'une monumentalisation du Nom propre, une épitaphe avec inscription ultime, bref, une mise en texte qui serait un peu, à sa manière, une mise en terre, mais du corpus cette fois. L'opération est double: retournement du sol, de l'humus, d'une part, érection et consolidation du socle, d'autre part. La plaque commémorative est désormais bien en place pour la postérité, pour mémoire, comme on dit: les derniers textes peuvent dès lors être promus au titre de testament - «la Conférence inachevée est sans contredit le testament littéraire de Ferron", lit-on sur la couverture -, l'inventaire du legs peut commencer.

Mais, peut-on aussi se demander, quelle est la transaction 
qui a cours ici autour du nom de Ferron, et d'abord, est-il justifié de parler de stratégie? Sans vouloir mener une lecture très détaillée ou encore moins systématique du paratexte ${ }^{6}$ entourant les deux textes proprement dits, je voudrais surtout m'attacher ici à quelques éléments du péritexte - couvertures, titres, mentions du genre, pages intérieures précédant le texte, tables, prière d'insérer - qui me paraissent significativement conduire la lecture vers une telle interprétation et permettent de répondre affirmativement à la question posée.

Ainsi, si, comme moi, vous entamez votre lecture en commençant par la fin, soit par la page quatre de couverture où se trouve le prière d'insérer par lequel tout livre tente l'exercice difficile de son auto-présentation, on remarquera que l'indice le plus révélateur de la mortification des ayants droit consiste sans aucun doute dans la multiplication des adjectifs que l'on trouvera, rhétorique oblige, en ce lieu livresque: "éclairage nouveau», "écrivain majeur», «humaniste et polémiste», «humilité touchante», «un Ferron bouleversé par la misère humaine», «préoccupé par le sort de ses semblables", "dénonciation la plus émouvante», "sort terrible», etc. Aucune ironie n'est, bien entendu, de mise dans ce contexte, le ton est grave, deadly serious. Comment douter que nous sommes devant une image, dans ce cas encore plus imaginaire que toute autre? Barthes disait dans Roland Barthes par lui-même d'«un rapport qui s'adjective (qu'il était) du côté de l'image, du côté de la domination, de la mort'": les prières d'insérer des livres posthumes ne peuvent plus qu'ajouter le vernis glacé (gloss), la dernière glose au portrait final qui tient lieu de commentaire.

Mais retournons le livre et laissons cette image pour en lire une autre, celle qui est le plus visible: la couverture. La conférence inachevée présente une photo couleur (sans titre) de Michel Dubreuil, et elle se détache sobrement sur un fond gris. Ce n'est pas à première vue une photo très réussie au plan esthétique, à moins qu'on apprécie ces clichés touristiques d'un certain bon mauvais goût, où le photographe cherche justement à faire de la «belle» photo, en jouant exagérément de la lumière ou de son trépied, mais elle reste des plus fonctionnelles, remplissant son rôle d'illustration. En comparaison, la couverture du Désarroi - une oeuvre de Ginette Bouchard, photo d'un noir et blanc travaillé par des techniques anciennes (une ombre sépia adoucit, on aurait envie de dire amortit, le jeu des contrastes), extraite d'une série intitulée Palladium - est beaucoup plus lourde, plus «symbolique» aussi, et semble davantage souligner le titre que le contenu de l'ouvrage. La 
couverture se fait d'ailleurs insistante et, passant le seuil du livre, loin d'occuper verticalement seulement la moitié de la couverture (l'autre moitié consiste en une bande d'un bleu-violet où se détachent, en vert-de-gris, les titre et nom de l'éditeur), elle se retrouve à nouveau reproduite in extenso dans les pages intérieures deux et trois: l'illustration ici ne peut pas ne pas faire partie du paratexte. Dans les deux cas, on peut déjà dire que le ton est donné: à leur manière, ces deux couvertures sonnent le glas, si j'ose l'expression, et inscrivent les signes du deuil. Mais allons y voir de plus près et montrons comment ces images constituent déjà une lecture du texte, avant même que le lecteur en ait lu la première ligne.

La couverture de La conférence inachevée représente une vue d'un parc fermé par une haute grille: le plan perspectif d'une longue allée bordée d'arbres nus est saisi en contre-plongée et légèrement déstabilisé du point de vue de l'opérateur. On distingue, à travers les grilles entrouvertes, mais retenues par une chaîne - on ne serait pas autrement surpris de découvrir la mise en garde qui convient à ces lieux, No Trespassing, et on se prend à se demander quel mystérieux «Rosebud» ou mot de passe permettra de pénétrer en ce domaine où n'entre sans doute pas qui veut - , on distingue donc, tout au fond, la vague forme d'une statue ou d'une fontaine et, plus loin derrière, la masse grise d'un édifice imposant où brillent quelques lumières. Toute la scène baigne dans une lumière bleutée, tout à fait crépusculaire, ou sont-ce les toutes premières lueurs du jour qui se lève ${ }^{8}$ ? Impossible de le dire en toute netteté, c'est l'heure bleue, cette heure insaisissable où l'on perd, comme dans la folie, la notion du temps. Encore à l'extérieur des murs (la photo est prise extra-muros, du dehors), nous sommes aussi hors livre, à l'orée d'un roman gothique noir peut-être, ce genre où les femmes étaient déclarées folles et retenues prisonnières contre leur gré dans la plus haute tour, comme le cliché du genre (The Madwoman in the Attic) le suggère. Interdit et transgression: sans rien connaître encore du Pas de Gamelin, le lecteur sait qu'il sera question ici d'un univers concentrationnaire (prison, asile, hôpital psychiatrique: tous lieux d'enfermement). En outre, la grille qui clôt, tout en l'entrebâillant, l'enceinte de ce parc renvoie également au titre, puisque la "conférence» (de conferre, "porter (ferre) avec, rassembler») entretient quelque rapport avec l'idée de la circonférence (de circumferre, "faire le tour»), cette courbe plane fermée qui constitue la limite extérieure d'un cercle: les connotations découlant de la circularité et de l'encerclement se reportent donc à un autre niveau sur les textes de Ferron eux-mêmes rassemblés dans un recueil, où tous les points périphériques, les diverses voix "concertantes" seront 
rapportés à un même centre ${ }^{9}$ : la question de l'autobiographie et de la folie. (Incidemment, remarquons le paraxode: la folie, sujet par excellence de la marginalité et de l'exclusion, devient ici le principe même de la cohérence et de l'ordonnance de l'oeuvre.) Par ailleurs, le mot «inachevée" vient dans le titre, telle la grille de la photo, «entrouvrir» ce que la figure du cercle avait de trop fermé (je reviens plus loin sur cette question du titre).

$\mathrm{Si}$, en dépit d'un certain romantisme noir, la couverture de La conférence inachevée reste subordonnée au texte (elle ne déborde pas sa stricte fonction utilitaire d'illustration du lieu principal dont il sera question dans le livre), celle du Désarroi est beaucoup plus esthétique - au sens noble du terme: nous sommes devant une oeuvre d'art ${ }^{10}$ - et par conséquent plus complexe à déchiffrer, gardant un rapport métaphorique avec le texte (dans le cas précédent, l'image fonctionnait plutôt de façon métonymique). La couverture du Désarroi consiste en une image énigmatique, qui ne livre pas d'emblée son sens et frappe surtout par une certaine pose pathétique, liée de manière évidente au thème de la mort, que vient d'ailleurs souligner avec plus de force encore le titre. Ici encore, nul ne pénètre impunément dans cette "forêt de signes». La photo représente en effet une étrange forêt onirique, dont le sol est jonché de copeaux et de feuilles-pierres, on ne sait trop au juste quelle est cette matière en décomposition, l'humus qui recouvre la terre. On aperçoit, à travers l'emmêlement de troncs d'arbres (ou tout n'est-il ici que simulacre? Ces arbres, dont les troncs sont lisses ou tavelés de taches suspectes, sont bien peu «naturels» et semblent en tout cas atteints eux aussi d'une maladie mortelle), une impressionnante statue d'homme: nu et renversé sur le dos, il lève un bras et son visage vers le ciel. On le sent blessé à mort, dans une ultime imploration, il agonise. La pose et le titre reconduisent tous deux le signifiant majeur de cette correspondance (le désarroi comme désordre moral, trouble, angoisse), publiée de façon posthume par celui des deux correspondants qui survit à l'autre. Ce n'est pas un hasard si le prière d'insérer en page quatre de couverture soulève directement la question de la légitimité quant à cette décision de publier sans l'accord concerté des deux partis impliqués: «Jacques Ferron se serait-il, de son vivant, opposé à la publication de ces échanges de lettres entre lui et le psychanalyste de renom ${ }^{11}$ Julien Bigras, lettres écrites pendant une période de deux ans sans que l'un et l'autre ne se rencontrent jamais? On ne le saura jamais. " On peut penser que le fait d'avoir dû trancher de cet indécidable («On ne le saura jamais»), véritable cas de conscience qui résonne déjà comme un regret dans la répétition du mot «jamais», n'est pas sans entraîner quelque 
doute, voire culpabilité, devant l'exposition d'une scène appartenant à la vie privée, à l'intimité du sujet. Serait-ce aller trop loin que de suggérer que cette question irrésolue mais à laquelle on a néanmoins passé outre, laisse des traces en revenant hanter l'image fantomatique qui a été retenue pour la couverture du livre? De façon analogue, il est difficile de ne pas lire telle phrase du prière d'insérer - «Loin d'être un ouvrage impudique où seraient dévoilés certains aspects intimes de la vie de ces deux écrivains et médecins [...]» - comme une semblable dénégation. La couverture du Désarroi, avec sa pose douloureuse, désespérée presque (on pense à Prométhée sur son rocher), dit plus et mieux que le discours préfaciel lui-même ne pourrait le faire, ce qu'il en coûte de toucher au corps du mort, et d'encourir le risque de peut-être trahir les volontés qui auraient été les siennes «de son vivant». Car c'est bien de ce dispositif thanatographique qu'il s'agit dans cette correspondance de nature transférentielle, où Ferron prend aux yeux de Bigras la «statue» d'un père ${ }^{12}$, figure d'autorité en ce qui a trait à la question de l'écriture: moins rendre un hommage in memoriam ou contribuer modestement à la connaissance de l'oeuvre de Ferron par la publication de documents inédits, lettres et «textes publiés en annexe», qu'instituer, par ces «propos d'une valeur inestimable» un conflit des noms propres, et perpétrer une tentative d'annexion et d'emprise de la part du survivant.
Étymologiquement, le mot emprise, précise Bigras dans la «Préface», a un sens juridique des plus précis, il s'agit d'une atteinte à la propriété (ou à la vie) privée résultant d'un acte administratif illégal. Avec Ferron, il fut tout de suite entendu que nulle emprise, nulle maî- trise ne s'exerceraient ni d'une part ni de l'autre. Et ce sacré Ferron a tenu bon d'une main de fer. De l'aide précieuse qu'il m'a apportée dans le livre que j'écrivais sur la folie, il n'a jamais voulu que je fasse mention; cela a failli d'ailleurs le rendre agressif (Le désarroi, p. 9).

Comme pour la phrase soulignant la pudeur de cette correspondance - pudeur qui, en attirant ainsi l'attention sur elle-même, devient paradoxalement, par un retour d'affect, le signal même de l'impudeur - , cet aveu de Bigras laisse songeur, car il peut aussi se lire à l'envers et produit alors les effets contraires à ceux qui étaient escomptés: c'est alors sa propre entreprise d'agression "atteinte à la propriété (ou à la vie) privée» -, d'«effraction ${ }^{13}$ " même, qui est mise au jour dans des expressions ambiguës telles que "ce sacré Ferron» ou «tenu bon d'une main de fer». II semble bien par ailleurs que ce ne soit pas la première fois que Bigras ne 
respecte pas le souhait pourtant explicite de Ferron, puisque non seulement il dévoile à cette occasion la discrétion de Ferron («il n'a jamais voulu que je fasse mention (de son aide)»), mais il lui dédicacera, en dépit de ses réserves pourtant évidentes, son livre Ma vie, ma folie. On peut penser que cette scène trouve avec la correspondance une vertaine répétition générale. Quoi qu'il en soit, le prière d'insérer du Désarroi est fortement marqué par cette rhétorique de la transgression, indiscrétion redoublée, impudeur se dévoilant elle-même...

On le voit: dès la couverture de ces deux ouvrages, la stratégie éditoriale du posthume est mise en place. Mais une telle opération - consécration, sacralisation ("sacré Ferron", "questionnements essentiels, salutaires»), mythocratie ${ }^{14}$ - toute positive qu'elle se veuille, n'est pas toujours exempte de négativité et elle peut aussi donner lieu, malgré qu'elle en ait, à des détournements de sens, quand ce n'est carrément à des règlements de comptes. Ainsi, dans le cas du Désarroi, sous couvert d'honorer la mémoire de celui qui fut tout à la fois la muse, le «truchement» et le destinataire privilégié, c'est une autre image plus violente, beaucoup moins sereine, du travail du deuil qui émerge çà et là en filigrane dans les lieux paratextuels, histoire assez sauvage où il s'agit de s'incorporer l'autre, de le dévorer, de le faire disparaître une deuxième fois. Dans le cas de La conférence inachevée, on ne retrouve pas un tel coup d'éclat, mais il se produit là aussi un geste d'effacement qu'il convient de commenter, puisqu'il prend pour objet l'un des éléments les plus importants du paratexte, soit le titre même qui a été choisi par les éditeurs de l'ouvrage. On apprend en effet dans une note des éditeurs dans Le désarroi qu'un texte de Ferron déjà paru, «Le glas de la Quasimodo», et qu'il avait envoyé à Bigras, portait en sous-titre, écrit à la main: "D'un recueil qui s'intitulera Contes d'adieu ou Contes du pays perdu». Les éditeurs ajoutent: «Jacques Ferron avait pensé intituler ainsi le recueil qui paraîtra en 1987 sous le titre La conférence inachevée ${ }^{15}$. Dans leur propre note, les éditeurs se font des plus discrets au sujet de cette question, pourtant capitale, du titre de La conférence inachevée:

La conférence inachevée présente une quinzaine de textes réunis en 1982 par Jacques Ferron et sur lesquels il était à travailler au moment de sa mort. Sur la première page du manuscrit, quelques titres retenus par l'auteur: «le Pas de Gamelin», «la Conférence inachevée». Le premier ainsi que le deuxjème de ces 
titres sont fort éloquents; ils illustrent l'atmosphère qui a pu présider à la confection du présent recueil't

La question qui vient tout naturellement à l'esprit est la suivante: pourquoi les éditeurs ont-ils retenu les deux derniers titres, plutôt que "Contes d'adieu» et "Sornettes et contes du pays perdu» qu'ils jugent pourtant "fort éloquents" et révélateurs de l'état d'esprit de Ferron au moment de l'écriture du livre? Comme ils ne disent rien eux-mêmes des motifs qui les ont conduits à choisir un titre plutôt qu'un autre, nous sommes réduits à spéculer sur ces raisons, mais il paraît tout de même curieux qu'ils aient écarté les deux titres qui étaient déjà employés par Ferron à l'époque, lorsqu'il faisait circuler ces textes, et qui semblaient donc avoir sa préférence. Sans entrer ici dans un procès d'intention, on peut se demander ce qui l'a emporté d'un titre sur l'autre. Sans doute, La conférence inachevée a-t-il été retenu parce qu'il renvoie explicitement à la situation biographique posthume, en faisant allusion à l'interruption provoquée par la mort de Ferron. Par ailleurs, le titre est à la fois assez abstrait et précis pour suggérer plusieurs pistes de lecture, plus que Le pas de Gamelin qui est, lui, trop topique ou local, trop allusif. Mais ce sont là des justifications bien banales, et il faut faire un «pas au-delà», si l'on ose dire, pour voir à quel point ce titre en cache un autre: La conférence inachevée sonne bien à l'oreille parce que, en effet, il nous rappelle un autre titre, L'entretien infini de Maurice Blanchot, dont il pourrait être la variation, la «conférence» étant bien une forme d'entretien, une réunion où des personnes traitent d'un sujet commun, alors que les mots «inachevée» et «infini» appartiennent au même réseau sémantique. La conférence inachevée inscrit ainsi Ferron dans des préoccupations esthétiques toutes modernes, alors qu'il aimait surtout à se voir comme le dernier scribe de la tradition orale et a toujours pratiqué une écriture qui avait plus d'affinités avec celle du XVIII siècle qu'avec celle de la modernité. Ce livre déporte ainsi le livre de Ferron du côté d'un conception de l'oeuvre ouverte, fragmentaire, lieu de rassemblement mais non pour autant discours clos ou totalisant, aire de circulation de voix multiples. Sans que cette conception de l'oeuvre soit absolument incompatible avec celle de Ferron, il semble pourtant que le point final, la clôture définitive étaient davantage marqués dans un titre comme "Contes d'adieu", qui signait sans réplique l'arrêt de l'oeuvre, en dehors de toute idée de mort biographique de l'écrivain. Il semble aussi qu'un titre comme "Contes ${ }^{17}$ du pays perdu» aurait dû s'imposer, ne serait-ce que parce qu'il met ce dernier texte en rapport avec toute l'oeuvre, et tout particulièrement avec les Contes du pays incertain, induisant ainsi une lecture renouvelée d'un thème qui a pesé 
très lourd sur la réception de l'oeuvre de Ferron, et amorçant le passage, l'articulation, entre la question du pays-territoire, porteur des valeurs identitaires, et un paysage intérieur, imaginaire, ayant partie liée avec la folie, où tous les repères identitaires du sujet sont "perdus». Est-ce le mot "pays» qui, en ces temps postréférendaires, les aura éloignés de ce titre? II y avait là en tout cas une belle occasion de vraiment «jeter un éclairage nouveau sur la vie et l'oeuvre" de Ferron, mais le titre retenu contribue plutôt à masquer le réaménagement global qui a cours dans ce livre, lequel assure moins la «succession» de l'oeuvre en lui donnant «suite», qu'il ne la met en désordre, la «desarroye», en réorientant en profondeur son interprétation. Á miser sur la conférence et sur la circonférence du cercle, les éditeurs ont opté pour le titre le plus neutre, le plus juste à leurs yeux, mais en croyant ainsi «faire le tour» de l'oeuvre ferronnienne, ils sont peut-être aussi restés captifs d'un effet de signifiant déjà fortement appelé et «porté» par le nom propre de Ferron et circonscrivant à l'avance un parcours de l'oeuvre. Rester fidèle aux intentions dernières, ou les trahir: il semble que, dans le cas de tels contrats posthumes, on ne puisse éviter de faire l'un et l'autre.

1. Gérard Genette, Seuils, coll. «Poétique», Paris, Seuil, 1987, p. 11.

2. Jacques Ferron, La conférence inachevée. Le pas de Gamelin et autres récits, édition préparée par Pierre Cantin, Marie Ferron et Paul Lewis, Montréal, VLB éditeur, 1987, 238 p.

3. Julien Bigras et Jacques Ferron, Le désarroi. Correspondance, Montréal, VLB éditeur, 1988, 176 p. Comme j'ai déjà eu l'occasion de recenser ces deux ouvrages de Ferron, je ne dirai rien ici de leur valeur littéraire; voir «Grandeurs et misères de l'arrière-texte", Spirale, Montréal, no 72, septembre 1987, p. 3, et «De la correspondance comme objet volant non identifié», Spirale, no 86, mars 1989, p. 3.

4. Il faudrait ici dire un mot du rôle que la nouvelle revue de Jean-Claude Germain, Le Québec littéraire, fait jouer à Ferron que l'on retrouve, dans le panthéon universel et personnel de Germain, aux côtés... de Goethe, ni plus ni moins. Dans le premier numéro, une belle photo de Ferron, retouchée çà et là de couleurs «modernes» (tout à fait dans l'esprit d'une certaine esthétique warholienne), donne une image «revampée», assez séduisante de Ferron, mais le cliché coloré n'est pas sans évoquer aussi, retour de perversité, des chromos religieux beaucoup plus anciens. Quoi qu'il en soit, Ferron semble ici veiller, tel un saint nouvellement canonisé, sur les destinées de la littérature québécoise. Dans la publicité pour le deuxième numéro («Prenez quelques livres de plus!»), Ferron se retrouve de nouveau à l'affiche, jouxté à La petite noirceur de Jean Larose et de quelques autres, littéralement dans la culotte d'un personnage bien ventru qu'on devine être Germain: belle image d'incorporation...

5. Rien n'indique en effet, à partir de l'appareil de notes réduit qui accompagne l'édition de La conférence inachevée, que les éditeurs aient cherché d'aucune façon à attirer l'attention sur leur présentation des textes. Cependant, dans une brève note, les éditeurs de la correspondance, Marie Ferron, Pierre Cantin et Julien Bigras, avoueront dans un même souffle qu'ils «ont colligé les 
lettres et les textes, les ont corrigés et annotés», sns préciser plus avant de quelle nature ont été ces corrections. Ils poursuivent en assurant le lecteur que «la correspondance n'en reste pas moins authentique et intégrale», mais ils nous informent ailleurs, avec une belle simplicité, que "cette lettre a été coupée car elle était trop longue» (Le désarroi, p. 70).

6. J'exclus du cadre de cette brève analyse tout ce qui concerne ce que Genette appelle l'épitexte (support médiatique, interviews, entretiens, etc.) et qui, associé à la description du péritexte, permet de donner une image complète du paratexte. Dans le cas d'ouvrages posthumes comme ceux-ci, l'épitexte doit aussi intégrer la parole de ceux qui parlent au nom de Ferron ou de son oeuvre: la critique. Dans le cas de la correspondance avec Bigras, c'est le livre tout entier qui pourrait être considéré comme épitexte.

7. Roland Barthes, Roland Barthes par lui-même, coll. «Écrivains de toujours", Paris, Seuil, 1975, p. 47.

8. "Chaque matin, avec une hâte déraisonnable, quelque peu folle, je m'amenais à Saint-Jean-de-Dieu tôt, très tôt, avant la fin du service de nuit, et ce sera, durant seize mois, toujours selon le même rituel obsessif que je franchirai le pas de Gamelin, à la faveur de l'obscurité ou dans la pâleur spectrale du petit jours (La conférence inachevée, p. 28).

9. Cette opération de recentrement du sujet est encore plus explicite dans le prière d'insérer: «[...] Jacques Ferron n'hésite pas à puiser à même sa vie et sa pratique médicale, à se situer au centre de son oeuvre [...]”.

10. Et qui plus est, d'une oeuvre-installation qui, comme certaines oeuvres postmodernes récentes, joue à fond la référence classique: la série dont est tirée cette oeuvre de Ginette Bouchard s'intitule Palladium (1986), nom dérivé de l'architecte italien Palladio, grand maître de la synthèse des classicismes grec et romain, dont l'art se caractérise par "une utilisation d'idiomes classiques hors de leurs contextes". II fut la référence suprême du néoclassicisme au XVIII siècle, celui qui «sut adapter l'antique aux formules de la vie contemporaine" (Petit Robert 2). Ce rapport à l'«idiome classique" n'est certes pas étranger à l'oeuvre de Ferron.

11. Le "renom" peut aussi s'entendre comme le redoublement du nom. On peut en voir les effets dans des lieux paratextuels: sur la page où l'on fait mention des ouvrages déjà parus des deux auteurs, ceux de Bigras sont décrits de façon inhabituellement détaillée, avec toutes les éditions, remaniements, etc., alors que ceux de Ferron sont signalés par leur seul titre (négligence de l'éditeur?); dans la table, l'alternance des textes reproduits en annexe - qui, soit dit en passant, compte pour plus du tiers du volume total - cherche à donner l'impression d'une répartition égale, mais de fait, ici comme ailleurs, c'est Bigras qui l'emporte dans la disposition finale et qui garde, comme dans la correspondance elle-même, le dernier mot.

12. Tout au long de la correspondance, Ferron ne cessera de se défendre contre Bigras, se méfiant du récit des origines qu'il est censé lui avoir inspiré ( "J'aurais dû vous dire (inutilement d'ailleurs car vos idées à ce propos étaient déjà faites) ma méfiance de vos origines" (p. 75)), cherchant à se retirer du rôle qu'il tente de lui faire tenir: «Et mon idée du père aboutit à ceci: on ne lui règle jamais son compte parce que les fils, beaucoup plus forts, le rejettent en arrière et se battent entre eux. On ne le tire pas car déjà il est mort et ne fait que chuchoter comme dans Hamlet: “Venge-moi - Oui, vieille taupe». Et c'est la mère qui sera tuée" (Le désarroi, p. 76-77).

13. "Je ne voudrais surtout pas m'introduire par effraction dans votre maison ou dans vos pensées" (Le désarroi, p. 73).

14. On constatera que, comme dans les cas d'Hubert Aquin, de Claude Gauvreau ou d'Émile Nelligan, la condition pour accéder à cette consécration mythocrati- 
que tient essentiellement à un certain rapport à la folie: ces deux ouvrages posthumes de Ferron remplissent, bien entendu, parfaitement cette condition.

15. Le désarroi, p. 106.

16. La contérence inachevée, p. 225. C'est moi qui souligne.

17. Malgré une insistance marquée à deux reprises par Ferron lui-même pour rattacher ces textes au genre des contes, on a indiqué la mention générique "et autres récits" sur la couverture du livre: manière de «moderniser», de retoucher ici encore son image en tant qu'écrivain? 